\title{
Cirugía de Fontan: experiencia inicial y resultados tempranos
} Reporte de un caso

\author{
Reyes Pavón Rodrigo*, Medina Reyes Daniel*, Romo Torres Ashley Aline*,
} Aguilar Alaniz Elizabeth**

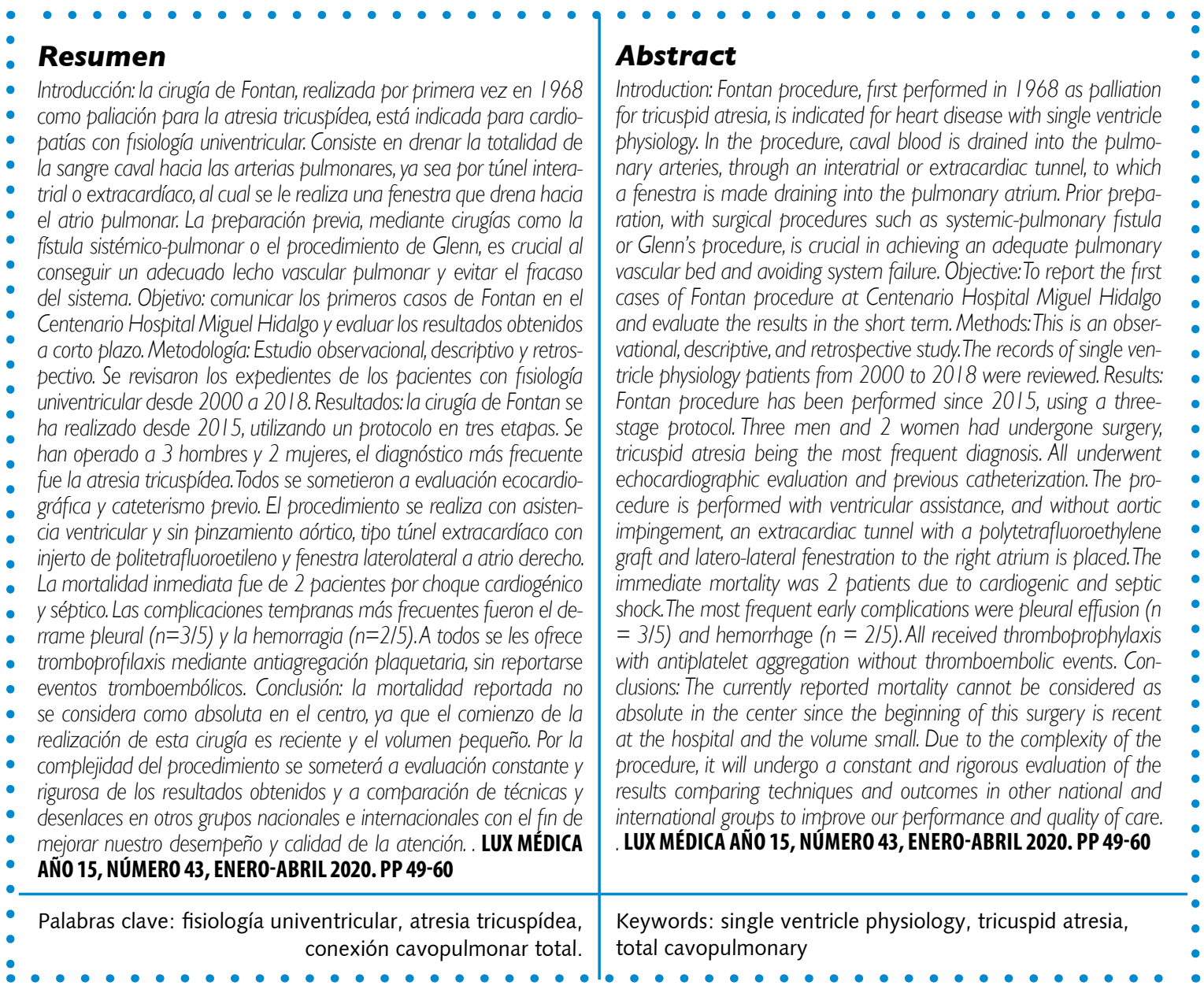

En honor del primera aniversario luctuoso del Dr. F. Fontan

"Most of the children with tricuspid atresia have a poor prognosis and die rather early (...) but we are of the opinion that they could profit from this corrective procedure..." (Francis Fontan sobre la reparación quirúrgica de la atresia tricuspídea1)

* Estudiantes del noveno semestre de la carrera de medicina del Centro de Ciencias de la Salud de la Universidad Autónoma de Aguascalientes.

** Jefe del Departamento de Cirugía Cardiotorácica, Departamento de Cirugía Cardiotorácica del Centenario Hospital Miguel Hidalgo, Aguascalientes, México.

Fecha de recibido: 4 de abril 2019

Fecha de aceptación: 4 de agosto 2019

Correspondencia: Dra. Elizabeth Aguilar Alaniz. Departamento de Cirugía Cardiotorácica. Centenario Hospital Miguel Hidalgo. Avenida Gómez Morín, La Estación, Alameda. Código postal 20259. Aguascalientes, Aguascalientes, México. Teléfono: +55 44999467 20. Correo electrónico: eaguilar12598@gmail.com. 


\section{Introducción}

La cirugía de Fontan-Kreutzer fue reportada por primera vez en 1971 (pero realizada en 1968) por Fontan como un procedimiento paliativo para la atresia tricuspídea. El procedimiento consistía en drenar la totalidad de la sangre de la vena cava superior hacia las arterias pulmonares empleando el procedimiento de Glenn, reportado previamente, asociado a la anastomosis término-terminal del extremo de la arteria pulmonar derecha al atrio derecho por uno homoinjerto valvular aórtico y el cierre del defecto septal atrial, la colocación de un homoinjerto valvular pulmonar en la vena cava inferior y la ligadura del tronco arterial pulmonar. ${ }^{1}$

A su vez, en 1973 Kreutzer describió un procedimiento similar de anastomosis atriopulmonar, pero sin colocar el homoinjerto valvular en vena cava inferior ni la anastomosis de Glenn. ${ }^{2}$ De esta manera, el atrio derecho hipertrófico era la bomba del circuito. Por su parte, Björk y cols. ${ }^{3}$ comprobaron que no era necesaria la presencia de válvulas en el circuito. De Leval y cols. ${ }^{4}$ encontraron, por estudios hemodinámicos, que la pulsación de este circuito generaba turbulencia, incrementando la resistencia al flujo anterógrado, por lo que propusieron la conexión cavopulmonar total mediante un túnel intraatrial que conducía la sangre caval inferior hacia la arteria pulmonar.

Finalmente, Marcelletti y cols. ${ }^{5}$ realizaron la interposición de un conducto extracardíaco entre la vena cava inferior y la arteria pulmonar, lo que mejoraba la función ventricular y vascular pulmonar, evitaba arritmias (por menor estrés parietal y reducción del tamaño del atrio derecho, así como menos líneas de sutura) y prevenía la turbulencia y estasis del flujo sanguíneo (al evitar la irregularidad del circuito). Además se podía realizar a corazón latiendo, sin necesidad de pinzamiento aórtico evitando, por tanto, isquemia miocárdica. ${ }^{6,7}$ Otra modificación propuesta fue la realización de una fenestra que permitiera un cortocircuito de derecha a izquierda (paso de sangre sistémica hacia el atrio pulmonar), lo cual mejoraba la precarga, reducía la presión venosa sistémica y prevenía la disfunción ventricular. ${ }^{8}$ Esto redujo la incidencia de derrame pleural posquirúrgico y el tiempo de estancia hospitalaria. ${ }^{9}$

La circulación de Fontan impone cambios circulatorios sistémicos, pulmonares y ventriculares. Se forma un cuello de botella determinado por la presión por arriba y abajo del cuello (la presión venosa sistémica y pulmonar) y la resistencia dentro del sistema. El ventrículo ya no controla el gasto cardiaco y el aumento en la contractilidad no aumenta el gasto, ya que la precarga está limitada; por lo tanto, tampoco es capaz de disminuir la congestión sistémica, únicamente bombea el gasto que el circuito de Fontan permite. La presencia de una fenestra mejora el gasto cardiaco al disminuir la congestión venosa, esto sacrifica cierto grado de saturación arterial. 
Un Fontan que fracasa se caracteriza por resistencias altas del sistema con bajo gasto y aumento considerable de presiones venosas. En cuanto a los cambios ventriculares, previo a la paliación, el ventrículo maneja volúmenes elevados que llevan a hipertrofia y dilatación, después de la cirugía se reduce drásticamente el volumen que recibe, lo cual ocasiona una disminución en la precarga $y$, por vasoconstricción arterial, un aumento en la poscarga. Si hay hipoflujo pulmonar después del nacimiento, existirá la necesidad de un procedimiento paliativo inicial (fístula) que permita "sobreflujo" y tiempo suficiente para el crecimiento del lecho vascular pulmonar, sin sobrecargar de volumen al ventrículo. ${ }^{10,11,12}$

La cirugía de Fontan-Kreutzer es actualmente considerada el abordaje para cardiopatías congénitas con un solo ventrículo funcional. Las indicaciones incluyen atresia tricuspídea, atresia mitral, síndrome de corazón izquierdo hipoplásico, ventrículo derecho hipoplásico, atresia pulmonar con septo interventricular intacto, doble salida del ventrículo derecho con atresia aórtica o defecto septal atrioventricular no balanceado. ${ }^{14}$

\section{El protocolo más aceptado actualmente incluye las siguientes etapas ${ }^{14}$ :}

I En la infancia temprana se valora un procedimiento paliativo: bandaje arterial pulmonar o fístula sistémica, esto depende de si la malformación está asociada a hipoflujo o a un flujo pulmonar aumentado.

II A los 6 meses se evalúan las arterias pulmonares y se realiza un Glenn bidireccional.

III Previo cateterismo, al año de la ciriugía de Glenn bidireccional, o entre los 2-4 años de edad, se completa el Fontan (si se realizó una fenestra de entre $4-6 \mathrm{~mm}$, se valora el cierre entre 6 y 12 meses después de completar el Fontan).

El objetivo de este artículo fue presentar los primeros cinco casos de cirugía de Fontan realizados en un centro de referencia regional y analizar los resultados obtenidos al inicio de la curva de aprendizaje del procedimiento.

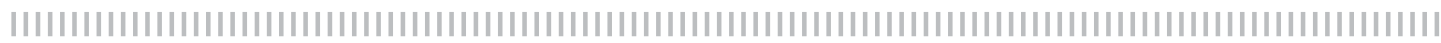

\section{Metodología}

Se realizó un estudio observacional, descriptivo y retrospectivo. Se revisaron de manera retrospectiva los expedientes de los pacientes con diagnósticos de fisiología univentricular que se sometieron a cirugía paliativa tipo Fontan en el periodo comprendido entre 2000 a 2018 en el Centenario Hospital Miguel Hidalgo y se describieron sus características sociodemográficas, prequirúrgicas, transquirúrgicas y posquirúrgicas inmediatas. 


\section{Resultados}

En el 2015 se inició la casuística de la cirugía de Fontan en el Centenario Hospital Miguel Hidalgo (CHMH) mediante el protocolo de 3 etapas, con modificaciones según las características clínicas (Tabla 1). Hasta 2018 se habían realizado 5 conexiones totales cavopulmonares, 3 de ellas en hombres y 2 en mujeres. Los diagnósticos fueron: atresia tricuspídea (3 pacientes, tipo IA en dos de ellos y IB en uno), ventrículo derecho hipoplásico con estenosis pulmonar severa) y doble entrada a ventrí- culo izquierdo con doble salida del ventrículo derecho y atresia valvular pulmonar.

Dos pacientes se sometieron a una paliación mediante fístula sistémico-pulmonar (Blalock-Taussig), tras la cual a uno de ellos se le realizó Glenn bidireccional, dos pacientes pasaron a Glenn bidireccional inicialmente y un solo paciente se sometió a cirugía de Fontan directamente. Cabe mencionar que todos se sometieron a evaluación ecocardiográfica y por cateterismo previa y todos se encontraban en ritmo sinusal previo a la cirugía (Tabla 2 ).

\section{Tabla I}

\section{Características sociodemográficas y clínicas de los pacientes}

\section{DATOS CLÍNICOS}

\begin{tabular}{|c|c|c|c|c|c|}
\hline Paciente & $\mathbf{1}$ & $\mathbf{2}$ & $\mathbf{3}$ & $\mathbf{4}$ & $\mathbf{5}$ \\
\hline Sexo & $\mathrm{M}$ & $\mathrm{M}$ & $\mathrm{F}$ & $\mathrm{F}$ & $\mathrm{M}$ \\
\hline $\begin{array}{c}\text { Edad al } \\
\text { diagnóstico }\end{array}$ & 4 meses & 10 años & 6 años & 22 días & 1 año \\
\hline $\begin{array}{c}\text { Diagnóstico } \\
\text { Atresia } \\
\text { IA y VDH }\end{array}$ & $\begin{array}{c}\text { Atresia } \\
\text { tricuspídea IB } \\
\text { (CIV restrictiva y CIA) }\end{array}$ & $\begin{array}{c}\text { VDH y Estenosis } \\
\text { Pulmonar Severa }\end{array}$ & $\begin{array}{c}\text { Atresia tricuspídea } \\
\text { IA (PCA) }\end{array}$ & $\begin{array}{c}\text { Doble Entrada VI } \\
\text { y Doble Salida del } \\
\text { VD, con Atresia } \\
\text { Pulmonar }\end{array}$ \\
\hline $\begin{array}{c}\text { Paliación primera } \\
\text { etapa }\end{array}$ & FBT & N/A & N/A & N/a & FBT \\
\hline Edad (años) & 4 meses & N/A & N/A & N/a & 1 año \\
\hline $\begin{array}{c}\text { Paliación Segun- } \\
\text { da Etapa }\end{array}$ & $\begin{array}{c}\text { Glenn } \\
\text { Bidireccional }\end{array}$ & N/A & Bidireccional & Bidireccional & N/A \\
\hline Edad (años) & 2 años & N/A & 6 años & 1 año 11 meses & N/A \\
\hline
\end{tabular}

$M=$ masculino, $F=$ femenino, $V D H:$ ventrículo derecho hipoplásico, SIV: septo interventricular, CIV: comunicación interventricular, CIA: comunicación interauricular, PCA: persistencia del conducto arterioso, VI: ventrículo izquierdo, VD: ventrículo derecho, $F B T=$ fistula sistémico pulmonar de Blalock-Taussig, N/A= no aplica . 


\section{Tabla 2}

\section{Características Prequirúrgicos a la cirugía de Fontan-Kreutzer}

\section{DATOS PREQUIRÚRGICOS}

\begin{tabular}{|c|c|c|c|c|c|c|}
\hline \multicolumn{2}{|c|}{ Paciente } & 1 & 2 & 3 & 4 & 5 \\
\hline \multicolumn{2}{|c|}{ Ritmo } & Sinusal & Sinusal & Sinusal & Sinusal & Sinusal \\
\hline \multicolumn{2}{|c|}{ Clase NYHA } & II & III & III & III & III \\
\hline \multicolumn{2}{|c|}{ Saturación 02 (\%) } & 90 & 81 & 91 & 90 & 67 \\
\hline \multirow{4}{*}{ ECO } & PAMP (mmHg) & 12 & 9 & 11 & 12 & 14 \\
\hline & PD2VI & 8 & 8 & 10 & 10 & 19 \\
\hline & FEVI (\%) & 76 & 80 & 74 & 67 & 74 \\
\hline & Válvula A/V & $\begin{array}{c}\text { Mitral sin altera- } \\
\text { ciones }\end{array}$ & $\begin{array}{l}\text { Mitral sin altera- } \\
\text { ciones }\end{array}$ & $\begin{array}{c}\text { Sin alteracio- } \\
\text { nes }\end{array}$ & $\begin{array}{c}\text { Mitral sin altera- } \\
\text { ciones }\end{array}$ & Sin alteraciones \\
\hline \multirow{4}{*}{ Angio-grafía } & Índice McGoon & 1.76 & 1.8 & 2.2 & 2.4 & 2 \\
\hline & Índice Nakata & 281 & 231 & 232 & 380 & 281 \\
\hline & PAMP (mmHg) & 12 & 9 & 11 & 12 & 14 \\
\hline & $\begin{array}{c}\text { Ramas Pulmo- } \\
\text { nares (mm) }\end{array}$ & $\begin{array}{c}\text { RD: } 11.5 \\
\text { RI: } 8.8 \\
\text { Confluentes, sin } \\
\text { estenosis }\end{array}$ & $\begin{array}{c}\text { RD: } 12 \\
\text { RI: } 13.2 \\
\text { Confluentes, sin } \\
\text { estenosis }\end{array}$ & $\begin{array}{c}\text { RD: } 12.6 \\
\text { RI: } 10.5 \\
\text { Confluentes, } \\
\text { sin estenosis }\end{array}$ & $\begin{array}{c}\text { RD: } 13.4 \\
\text { RI: } 12.3 \\
\text { Confluentes, sin } \\
\text { estenosis }\end{array}$ & $\begin{array}{c}\text { RD: } 13.7 \\
\text { RI: } 8.5 \\
\text { Confluentes, sin } \\
\text { estenosis }\end{array}$ \\
\hline
\end{tabular}

Clase NYHA= clase funcional de la New York Heart Association modificada por Ross. ECO= ecocardiograma, $P A M P=$ presión arterial media de la arteria pulmonar $P 2 V I=$ presión telediastólica del ventrículo izquierdo, $F E V I=$ función ventricular izquierda, Válvula $A / V=$ válvula atrio-ventricular, $R D=$ rama pulmonar derecha, RI= rama pulmonar izquierda.

La técnica quirúrgica consiste en esternotomía media, asistencia con circulación extracorpórea sin pinzamiento aórtico, Fontan extracardíaco con injerto de politetrafluoroetileno $(18 \mathrm{~mm}$ de diámetro en los 5 casos), realización de fenestra rutinaria por anastomosis laterolateral de injerto a atrio derecho (entre $7-8 \mathrm{~mm}$ diámetro) y colocación rutinaria de marcapasos epicárdico. Cabe mencionar que no se contó con cateterismo postoperatorio en ningún caso
(Tablas 3 y 4). La mortalidad temprana (a 30 días) del centro es del $40 \%(n=2)$. El protocolo del centro incluye tromboprofilaxis con antiagregación plaquetaria después de la fístula sistémico-pulmonar, el procedimiento de Glenn y el procedimiento de Fontan. En este último se emplea ácido acetilsalicílico a dosis de $100 \mathrm{mg}$ al día, sin reportarse eventos tromboembólicos o reingresos por hemorragia con este régimen. 


\section{Tabla 3}

Características durante la realización de la cirugía de Fontan-Kreutzer

\section{DATOS TRANSQUIRÚRGICOS}

\begin{tabular}{|c|c|c|c|c|c|}
\hline Paciente & $\mathbf{1}$ & $\mathbf{2}$ & $\mathbf{3}$ & $\mathbf{4}$ & $\mathbf{5}$ \\
\hline $\begin{array}{c}\text { Edad al Fontan } \\
\text { (años) }\end{array}$ & 12 & 11 & 10 & 5 & 5 \\
\hline $\begin{array}{c}\text { Intervalo } \\
\text { Paliación-Fontan } \\
\text { (años) }\end{array}$ & 10 & 1 & 5 & 4 & 5 \\
\hline $\begin{array}{c}\text { Peso al Fontan } \\
\text { (kg) }\end{array}$ & 33 & 81 & 24 & 20 & 8 \\
\hline $\begin{array}{c}\text { Fenestración } \\
\text { (mm) }\end{array}$ & 8 & 188 & 100 & 7 & 158 \\
\hline $\begin{array}{c}\text { Tiempo de CEC } \\
\text { (min) }\end{array}$ & 188 & No & No & No & No \\
\hline \begin{tabular}{c} 
Complicaciones \\
\hline
\end{tabular}
\end{tabular}

$C E C=$ Circulación extracorpórea

\section{Tabla 4}

\section{Características posquirúrgicas inmediatas y complicaciones tempranas de la cirugía}

\section{DATOS POSQUIRÚRGICOS}

\begin{tabular}{|c|c|c|c|c|c|}
\hline Paciente & 1 & 2 & 3 & 4 & 5 \\
\hline Saturación O2 (\%) & 90 & 93 & 85 & 76 & 86 \\
\hline FEVI (\%) & 56 & 80 & 63 & 55 & 63 \\
\hline Derrame pleural & No & $\mathrm{SI}$ & $\mathrm{No}$ & $\mathrm{Si}$ & $\mathrm{Si}$ \\
\hline Tiempo con Toracostomía (días) & $\mathrm{N} / \mathrm{a}$ & 19 & $\mathrm{~N} / \mathrm{a}$ & 8 & $\begin{array}{c}15 \text { días y pleuro- } \\
\text { desis }\end{array}$ \\
\hline Sangrado & No & No & $\mathrm{Si}$ & $\begin{array}{c}\text { Si } \\
\text { Requirió } \\
\text { intervención }\end{array}$ & No \\
\hline Otras complicaciones & No & No & No & No & No \\
\hline Tiempo Intubación (h) & 24 & 48 & 48 & 192 & 24 \\
\hline Tiempo UTIP (días) & 5 & 3 & 3 & 8 & 1 \\
\hline Tiempo Estancia Piso (días) & 10 & 21 & 4 & N/a & 45 \\
\hline Defunción & No & No & $\begin{array}{c}\text { Si: Choque } \\
\text { cardiogénico }\end{array}$ & $\begin{array}{c}\text { Si: Choque } \\
\text { cardiogénico } \\
\text { y séptico }\end{array}$ & No \\
\hline Reingreso & No & No & N/a & N/a & No \\
\hline Último seguimiento & Asintomático & Asintomático, & Non sinusal. & N/A & Asintomático \\
\hline
\end{tabular}

FEVI= fracción de expulsión del ventrículo izquierdo. UTIP= unidad de terapia intensiva pediátrica. $N / a=$ no aplica 


\section{Caso 1}

Paciente del sexo masculino, diagnosticado a los 4 meses de edad con atresia tricuspídea IA y ventrículo derecho hipoplásico y se le realiza fístula sistémico-pulmonar de Blalock-Taussig; a los dos años de edad se le realizó Glenn bidireccional en otra institución y, finalmente, se completó el Fontan a los 12 años en nuestro hospital (figuras 1 a 4$)$.

\section{Caso 2}

Paciente del sexo masculino, diagnosticado a los 10 años con atresia tricuspídea IB, asociada a comunicación interventricular restrictiva (muscular, de $5 \mathrm{~mm}$ de diámetro, anillo aórtico de $21 \mathrm{~mm}$ de diámetro) y a comunicación interauricular amplia $(19 \mathrm{~mm}$ de diámetro), sin procedimientos paliativos, se realizó cirugía de Fontan a los 11 años.

\section{Caso 3}

Paciente del sexo femenino, diagnosticada a los 6 años con ventrículo derecho hipoplásico y estenosis pulmonar severa. Se sometió a los 6 años a cirugía de Glenn bidireccional en otra institución, se le dio seguimiento este hospital hasta los 10 años, cuando se decide completar el Fontan. Falleció a los 3 días posquirúrgicos, secundario a un choque cardiogénico.

\section{Caso 4}

Paciente del sexo femenino, diagnosticada a los 22 días de vida con atresia tricuspídea IA (asociada a conducto arterioso persistente); al año y once meses se realizó la paliación con cirugía de Glenn bidireccional, y a los 5 años se realizó la cirugía de Fontan (figura 5). Fallece a los 8 días posquirúrgicos por choque mixto, cardiogénico y séptico de foco pulmonar.

\section{Caso 5}

Paciente del sexo masculino, diagnosticado al año de vida con doble entrada a ventriculo izquierdo y doble salida del ventrículo derecho con atresia valvular pulmonar. Se le realizó paliación con fístula sistémicopulmonar de Blalock-Taussig y pasa a cirugía de Fontan a los 5 años de vida.

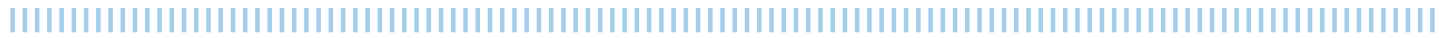

\section{Discusión}

La mortalidad temprana de la cirugía de Fontan en estudios reportados entre 1990 y 2009 varía entre el $0.5 \%$ y el $6 \%$, un claro descenso en comparación con el período entre 1980-1990, donde la mortalidad variaba entre el $4.3 \%$ y el $23 \%$. Asimismo, la supervivencia a 15 años ha aumentado hasta el $95 \%$, a 25 años se reporta del $83 \%$ y a 30 años del $43 \% .{ }^{16}$ En la actual serie de casos presentados, la mortalidad salta a la vista, sin embargo no consideramos este porcentaje de mortalidad como absoluto; sólo son los primeros 5 casos del hospital, la curva de aprendizaje y el volumen de pacientes operados será determinante en los resultados concluyentes.

Una revisión realizada por Akintoye y cols, $^{18}$ indica que, de 2001 a 2014 se reali- zaron 15,934 procedimientos de este tipo en Estados Unidos, con una mediana de edad a los 3 años. La patología que con más frecuencia fue sometida a cirugía de Fontan fue el síndrome de corazón izquierdo hipoplásico $(29 \%)$ seguido por la atresia tricúspide (15.8\%), lo que difiere de la serie que se presenta, donde la atresia tricuspídea es el diagnóstico más frecuente $(n=3 / 5)$.

Aunque el cateterismo ha sido considerado como una rutina prequirúrgica, recientemente se ha considerado limitar su realización a pacientes de alto riesgo. Prakash y cols $^{19}$ proponen un algoritmo (complementado por Yassin y $\operatorname{cols}^{20}$ ) donde la presencia de criterios clínicos, ecocardiográficos y por angiotomografía y/o angiorresonancia permiten clasificar al pa- 
ciente como con o sin criterios de riesgo. Mencionan como criterios clínicos de riesgo la presencia de enfermedad pulmonar crónica (o anormalidades en radiografía torácica $>3$ meses, o uso de oxígeno suplementario $>3$ meses), heterotaxia, síndrome genético, saturación de oxígeno $<76 \%$, o hemoglobina $>18 \mathrm{~g} / \mathrm{L}$. Como criterios ecocardiográficos la presencia de regurgitación moderada de la válvula atrioventricular, disfunción ventricular moderada ( $\mathrm{FE}<50 \%$ ), insuficiencia aórtica moderada, estenosis de la vena pulmonar (gradiente medio por Doppler $>3 \mathrm{mmHg}$ ), sospecha de colaterales aortopulmonares o septo interatrial restrictivo; $y$, finalmente, hallazgos por angiotomografía o angiorresonancia, como estenosis moderada (o discontinuidad) de la arteria pulmonar, estenosis de la vena pulmonar, o anomalía del retorno venoso sistémico.

Cualquiera de los criterios clínicos mencionados amerita cateterismo diagnóstico, su ausencia clasifica a un paciente como sin características de alto riesgo y puede proceder a cirugía sin necesidad de cateterismo previo. Sin embargo, Mohammad y cols, ${ }^{21}$ consideran que el cateterismo es necesario en todos los pacientes, ya que es el único método que provee información diagnóstica extra con certeza, como la presencia de colaterales pulmonares, estenosis arterial pulmonar (ambas no se comprueban adecuadamente por angiografía no invasiva) presión ventricular, presión en Glenn y resistencia vascular pulmonar. Además, permite una intervención terapéutica, como la embolización de las colaterales. En el $\mathrm{CHMH}$, dado que la casuística se inició recientemente, se realiza cateterismo a todos los pacientes considerando que es necesario adquirir mayor experiencia antes de omitir algún estudio preoperatorio de rutina.
La modificación más notoria que ha sufrido esta cirugía en los últimos años ha sido el cambio de elección de túnel lateral intracardiaco $(32.8 \%)$ a la colocación de un injerto cavopulmonar extracardiaco (44\%). ${ }^{16}$ La mortalidad y morbilidad reportadas con esta técnica son bajas, no se ha observado obstrucción del conducto y, además, se ha demostrado un crecimiento compensador ante el injerto por parte de la vena cava inferior y el diámetro de la arteria pulmonar. El diámetro óptimo del mismo varía entre 16-18mm para niños de 2-3 años, mayores diámetros resultan en un mayor volumen estancado en la fase espiratoria. ${ }^{22-24}$

Los principales reportes de este procedimiento en México proceden de los grupos de trabajo del Instituto Nacional de Cardiología "Ignacio Chávez" (INCICH) y del Hospital Infantil de México "Federico Gómez" (HIM). La serie más reciente del primero incluye 81 pacientes operados entre 1989 y 2003, y 53 pacientes del segundo en un periodo de 1983 a 2012. El diagnóstico más frecuente fue la atresia tricuspídea en ambos grupos. En el INCICH la edad más frecuente de realización corresponde a la de los menores de 5 años, con un rango de 1.85 a 22.7 años, para el HIM la edad varió de 0.6 a 16 años. En el INCICH fue más frecuente la realización de túnel intracardíaco (75\%), mientras que en el HIM solo al $24 \%$ se le practicó está técnica. La realización de fenestra fue más frecuente para el grupo del HIM (87\%) contra un $65 \%$ del INCICH. Finalmente, la mortalidad operatoria fue del $17.2 \%$ para el Instituto y del 34.3\% para el Hospital Infantil. En la Tabla 5 se resumen las principales complicaciones tempranas en ambos grupos de trabajo. ${ }^{25,26}$ 


\section{Tabla 5}

Comparación de las principales complicaciones posquirúrgicas que se presentaron en dos centros de alto volumen de cirugía de Fontan.

\begin{tabular}{|l|l|}
\hline \multicolumn{1}{|c|}{ Hospital Infantil de México “Federico Gómez” 26 } & Instituto Nacional de Cardiología “Ignacio Chávez” 25 \\
\hline & \\
Insuficiencia renal aguda (28\%) & Derrame pleural (100\%) \\
Complicaciones neurológicas (21.9\%) & Trastornos de la conducción (38\%) \\
Neumonía (15.6\%) & Ascitis significativa que requirió catéter de diálisis \\
Sepsis nosocomial (15.6\%) & peritoneal (22\%) \\
Derrame pleural y quilotórax (15.6\%) & Obstrucción de la anastomosis (16\%) \\
Choque cardiogénico (15.6\%) & Sangrado (15\%) \\
Arritmias (12.5\%) & Parálisis diafragmática (10\%) \\
Sangrado (9.4\%) & Derrame pericárdico (7\%) \\
& Evento vascular cerebral (2.4\%) \\
\hline
\end{tabular}

Es también parte del protocolo del Hospital Hidalgo la realización de la cirugía sin someter a derivación cardiopulmonar cuando es posible. Sobre esto, el grupo del INCICH evalúo la mortalidad entre pacientes sometidos a cirugía de Fontan con circulación extracorpórea y aquellos sin derivación. En el primer grupo se obtuvo una mayor mortalidad (5.2\% vs $0 \%$ ), sangrado $(26.9 \%$ vs $0 \%)$, menor fracción de expulsión y de acortamiento y mayor insuficiencia valvular, pero morbilidad similar en el mediano plazo. A pesar de la limitada casuística, se apoya el concepto de realizar Fontan sin derivación cardiopulmonar siempre que sea posible. ${ }^{27}$

\section{Conclusiones}

La cirugía de Fontan, como la mejor opción de procedimiento paliativo para pacientes con fisiología univentricular comienza su curva de aprendizaje en nuestra institución, por lo que la evaluación constante y rigurosa de los resultados obtenidos y la comparación de las técnicas empleadas y desenlaces que se logran en otros grupos de trabajo, tanto de México como internacionales, permitirá mejorar la calidad de atención ofrecida a los pacientes. La mayor dificultad radica en la correcta elección y preparación previa al Fontan de los pacientes, esto incluye la adecuada evaluación ecocardiográfica y hemodinámica, además de optimizar el tiempo quirúrgico cuando se utiliza derivación cardiopulmonar. Los resultados y complicaciones que surjan a largo plazo, así como la calidad de vida, son determinadas por la adaptación a los cambios hemodinámicos secundarios a la cirugía, tal como la verdadera mortalidad inmediata, quedan por ser evaluados a medida que se amplíe la serie de pacientes. 


\section{Bibliografía}

1. Fontan $F$, Baudet E. Surgical repair of tricuspid atresia. Thorax. 1971; 26: 240-248.

2. Kreutzer G, Galíndez E, Bono H, De Palma C, Laura J. An operation for the correction of tricuspid atresia. J Thorac Cardiovasc Surg.1973; 66: 613-621.

3. Björk, v. O., Olin, C. L., Bjarke, B. B. \& Thorén, C. A. Right atrial-right ventricular anastomosis for correction of tricuspid atresia. J Thorac Cardiovasc Surg. 1979; 77: 452-458.

4. de Leval MR, Kilner $P$, Gewillig $M$, et al: Total cavopulmonary connection: a logical alternative to atriopulmonary connection for complex Fontan operations. Experimental studies and early clinical experience. J Thorac Cardiovasc Surg. 1988; 96: 682-695.

5. Marcelletti C, Corno A, Gannico S, Marino B. Inferior vena cavapulmonary artery extracardiac conduit. A new form of right heart bypass. J Thorac Cardiovasc Surg. 1990; 100: 228-232.

6. Petrossian E, Thompson LD, Hanley FL. Extracardiac conduit variation of the Fontan procedure. Adv Card Surg. 2000; 12: 175-198.

7. Van Son JA, Reddy $M$, Hanley FL. Extracardiac modification of the Fontan operation without use of prosthetic material. J Thorac Cardiovasc Surg. 1995; 110: 1766-1768.

8. Bridges ND, Lock JE, Castaneda AR. Baffle fenestration with subsequent transcatheter closure: Modification of the Fontan operation for patients at increased risk. Circulation. 1990; 82: 1681-1689.

9. Bridges ND, Mayer JE, Jr, Lock JE, Jonas RA, Hanley $F L$, Keane JF, et al. Effect of baffle fenestration on outcome of the modified Fontan operation. Circulation. 1992; 86: 1762-1769.

10. Gewillig M, Brown SC, Heying R, Eyskens B, Ganame J, Boshoff $D E$, et al. Volume load paradox while preparing for the Fontan: not too much for the ventricle, not too little for the lungs Interact Cardiovasc Thorac Surg. 2010; 10: 262-265.

11. Gewillig $M$, Brown SC. The Fontan circulation after 45 years: update in physiology. Heart. 2016; 102: 1081-1086.

12. Jolley M, Colan SD, Rhodes J, DiNardo J. Fontan physiology revisited. Anesth Analg. 2015; 121: 172-82.

13. Gewillig M. The fontan circulation. Heart. 2005; 91 : 839-846.

14. Rao PS. Fontan operation: Indications, short and long term outcomes. Indian J Pediatr 2015; 82:1147-56.

15. Windsor J, Townsley MM, Briston D, Villablanca PA, Alegria JR, Ramakrishna F. Fontan palliation for singleventricle physiology: perioperative management for noncardiac surgery and analysis of outcomes. J Cardiothorac Vasc Anesth. 2017; 31: 2296-2303.

16. Kverneland L, Kramer P, Ovroutski S. Five decades of the Fontan operation: A systematic review of international reports on outcomes after univentricular palliation. Congenit Heart Dis. 2018; 13: 181-193.

17. Fontan F, Kirklin JW, Fernandez G, Costa F, Naftel DC, Tritto $F$, et al. Outcome after a "Perfect" Fontan operation. Circulation. 1990; 81; 1520-1536.

18. Akintoya E, Miranda W, Veldtman G, Connolly H, Egbe A, National trends in Fontan operation and inhospital outcomes in the USA. Heart. 2018; 0: 1-7.

19. Prakash $A$, Khan MA, Hardy $R$, Torres AJ, Chen $J M$, Gersony WM. A new diagnostic algorithm for assessment of patients with single ventricle before a Fontan operation. J Thorac Cardiovasc Surg. 2009;138: 917-923.

20. Yassin $H$, Bhat AN, Tysarowski $P$, Masud F, Dilawar $M$. Noninvasive evaluation of single-ventricle patients before Fontan operation. Asian Cardiovasc Thorac Ann. 2015:23:412-417

21. Mohammad Nijres B, Murphy JJ, Diab K, Awad S, Abdulla RI. Routine Cardiac Catheterization Prior to Fontan Operation: Is It a Necessity? Pediatr Cardiol. 2018; 39: 818-823.

22. Nakano $T$, Kadoa $H$, Tatewakia $H$, Hinokiyamaa $K$, Odaa S, Ushinohamab $\mathrm{H}$, et al. Results of extracardiac conduit total cavopulmonary connection in 500 patients. Eur J Cardiothorac Surg. 2015; 48: 825-832.

23. Iwaki $H$, Masuda $M$, Shiokawa $Y$, Imoto $Y$, Nakano $\mathrm{T}$, Sonoda $\mathrm{H}$ et al. The somatic growth of autologous vessels in veous pathway after extracardiac total cavopulmonary connection. J Card Surg 2008; 26:454458.

24. Itatani K, Miyaji K, Tomoyasu T, Nakahata Y, Ohara $\mathrm{K}$, Takamoto $\mathrm{S}$ et al. Optimal conduit size of the extracardiac Fontan operation based on energy loss and flow stagnation. Ann Thorac Surg 2009; 88:565-573.

25. Calderón J, Ramírez S, Viesca R, Ramírez L, Casanova M, García J, et al. Cirugía de Fontan. Factores de riesgo a corto y mediano plazo. Arch Cardiol Mex. 2005; 75: 425-434.

26. Bolio $A$, Ruiz $S$, Romero $P$, Hernández $G$, Villasís $M$ Pronóstico de niños cardiópatas sometidos a cirugía de Fontan: experiencia de treinta años en el Hospital Infantil de México Federico Gómez. Bol Med Hosp Infant Mex. 2013; 70: 151-158.

27. Ortiz M, Espinoza O, Ramírez S, Calderón J, García J, Cervantes J. Comparación de pacientes sometidos a cirugía de Fontan con y sin derivación cardiopulmonar. Arch Cardiol Mex. 2016; 86: 1-10. 


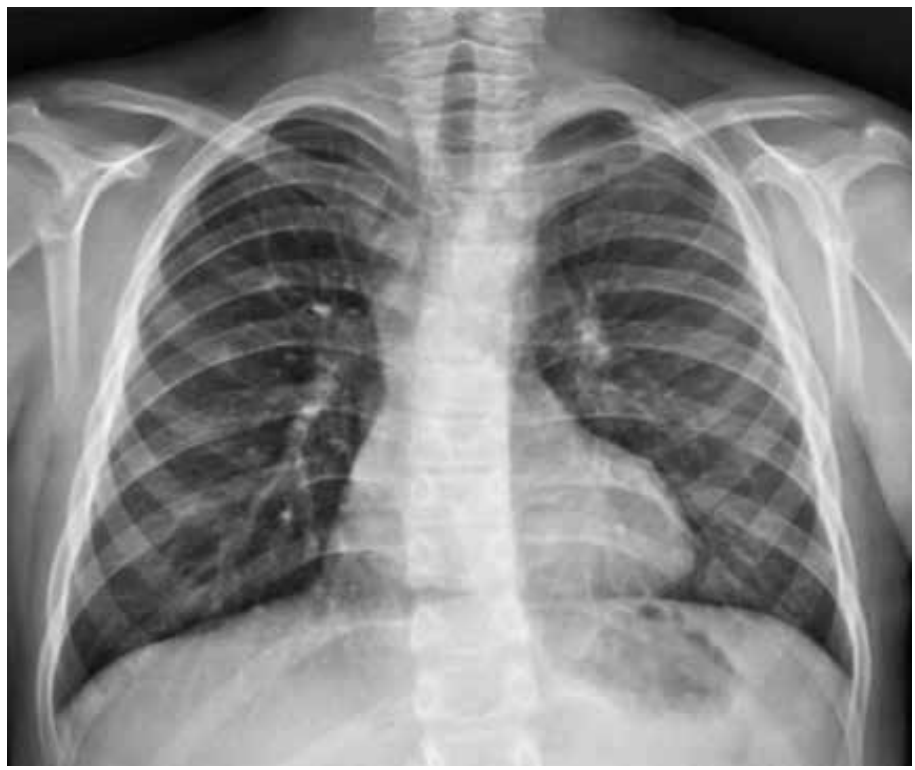

Figura 1. Radiografía de tórax en posteroanterior, prequirúrgica a la cirugía de Fontan. Cardiomegalia insignificante y arco medio excavado. Flujo pulmonar adecuado.

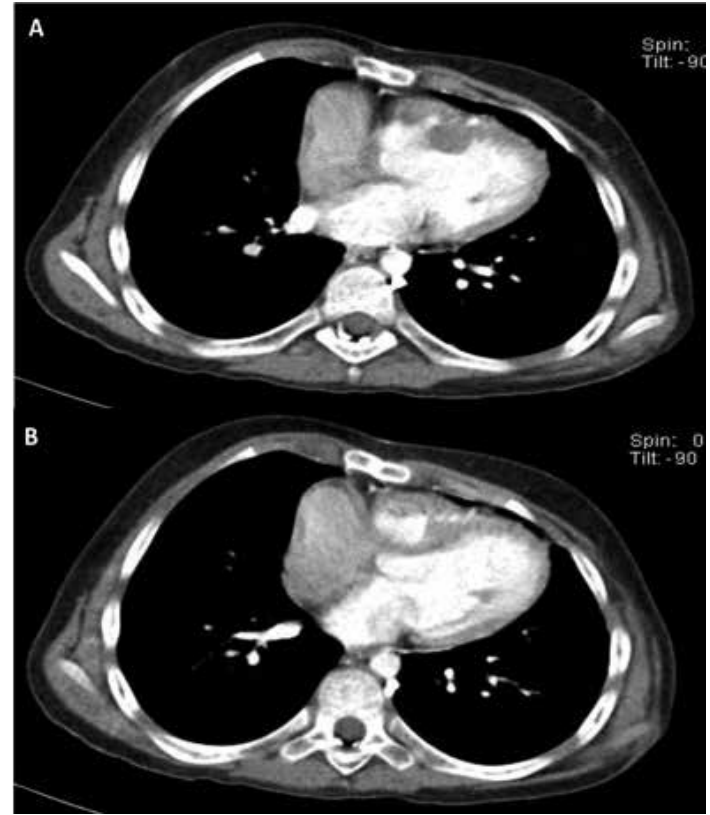

Figura 2. Angiotomografía contrastada en fase arterial, cortes axiales, realizada después de la cirugía de Glenn y antes de completar la conexión cavopulmonar, donde se observa el ventrículo derecho hipoplásico, en (A) ausencia de comunicación atrioventricular derecha, sustituida por banda de tejido hipodenso y ausencia de flujo anterógrado al ventrículo a través de la válvula tricúspide.

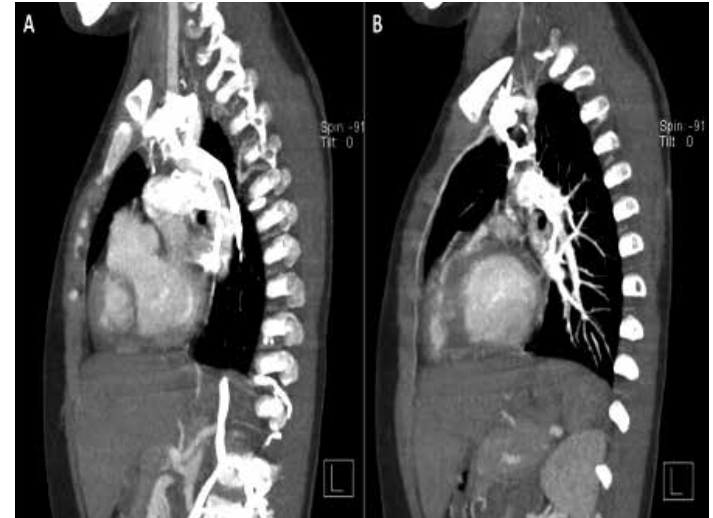

Figura 3. Angiotomografía contrastada en fase arterial, cortes sagitales, realizada después de la cirugía de Glenn y antes completar la conexión cavopulmonar. En (A) se observa el ventrículo derecho hipoplásico, a comparación del ventrículo izquierdo. En (B) se observa la atresia pulmonar con el infundíbulo hipoplásico. 


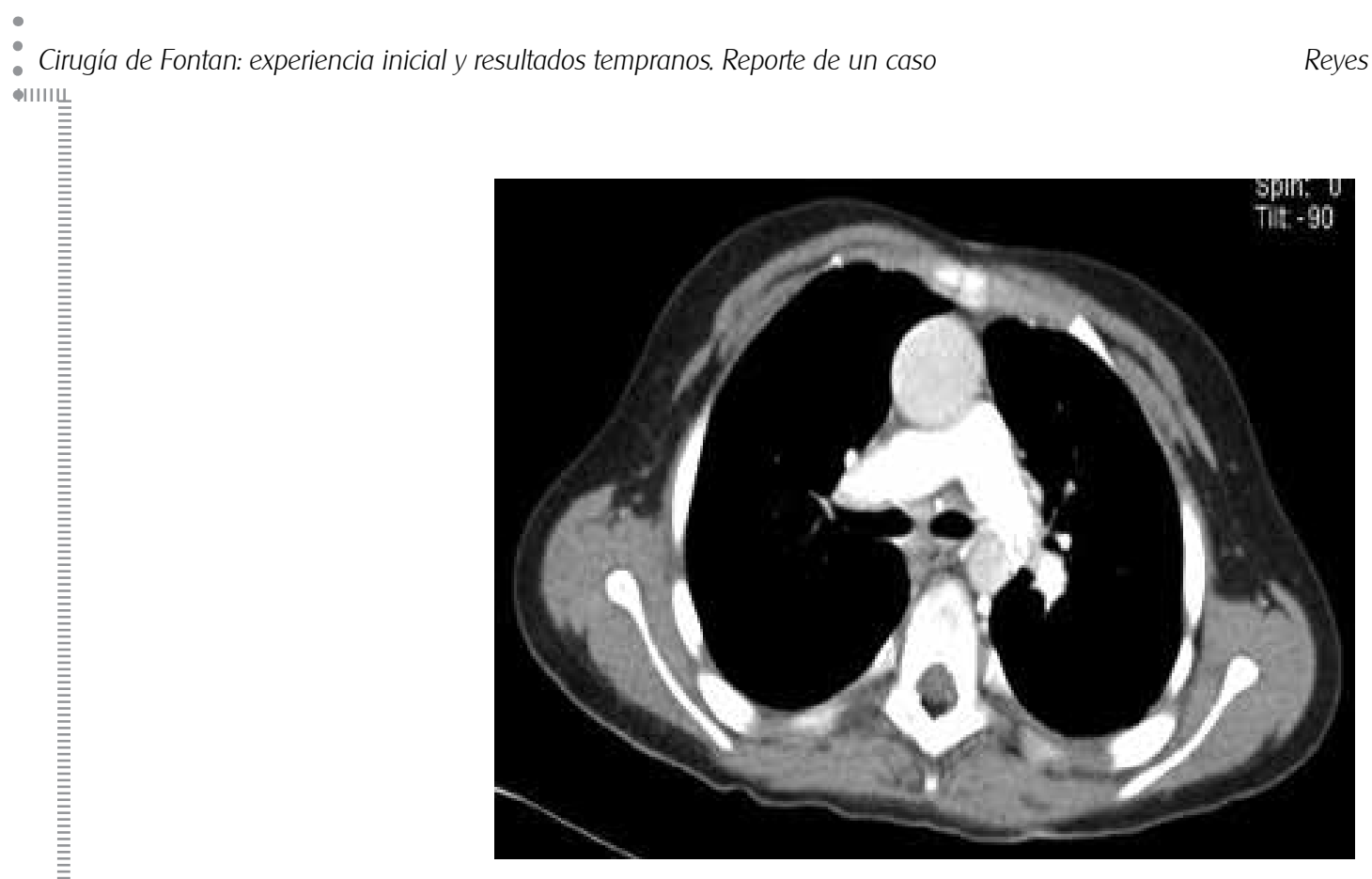

Figura 4. Angiotomografía contrastada en fase arterial, corte axial, realizada después de la cirugía de Glenn y antes de completar la conexión cavopulmonar, donde se aprecia adecuadamente la anatomía y diámetros de las ramas pulmonares confluentes.
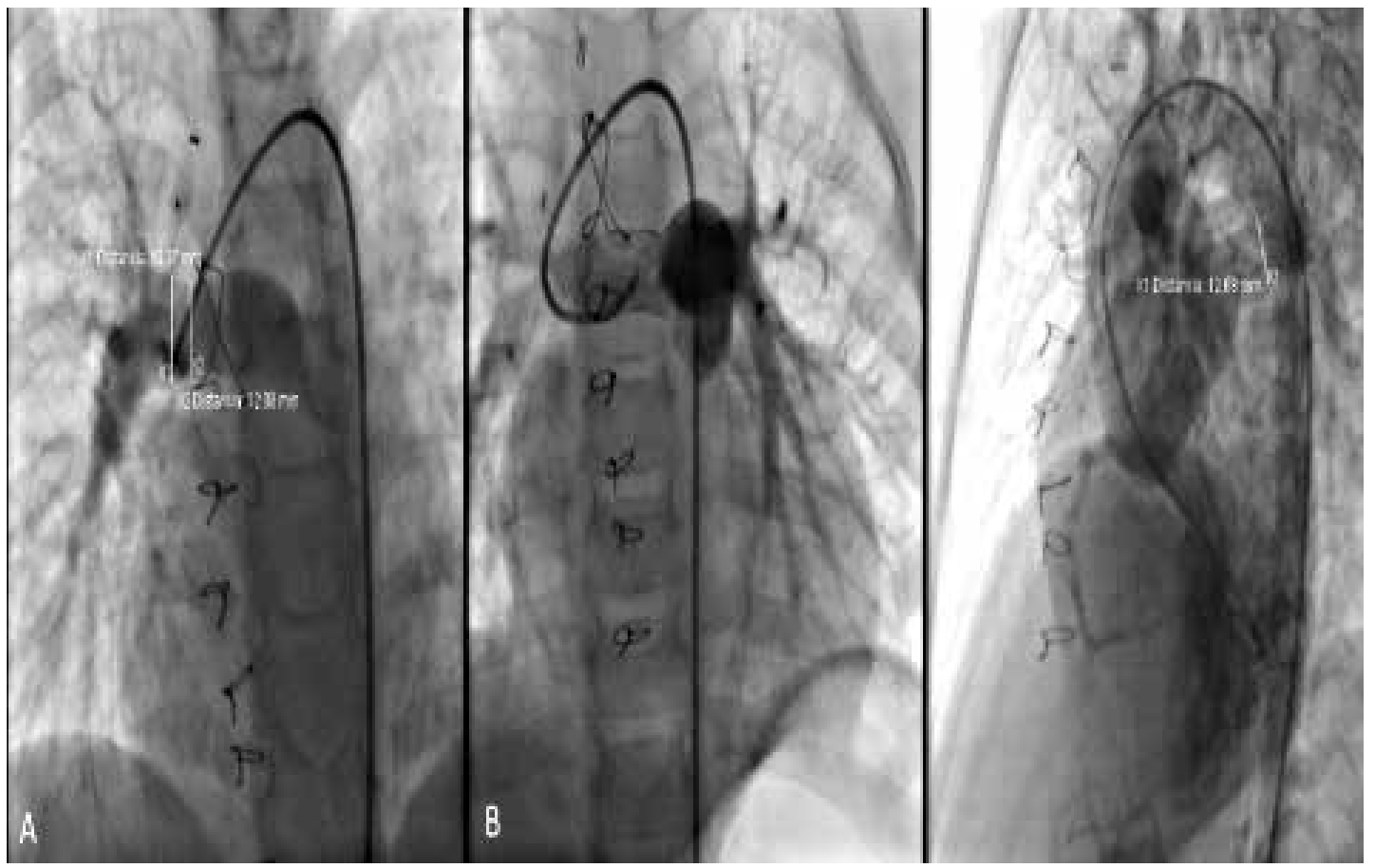

Figura 5. Angiografía diagnóstica previa a la cirugía de Fontan, donde se evalúa la anatomía de las ramas pulmonares. A) Diámetro de la rama pulmonar derecha. B) Ramas pulmonares confluentes. C) Diámetro de la rama pulmonar izquierda. 